\title{
Prediction of active ingredients in Salvia miltiorrhiza Bunge. based on soil elements and artificial neural network
}

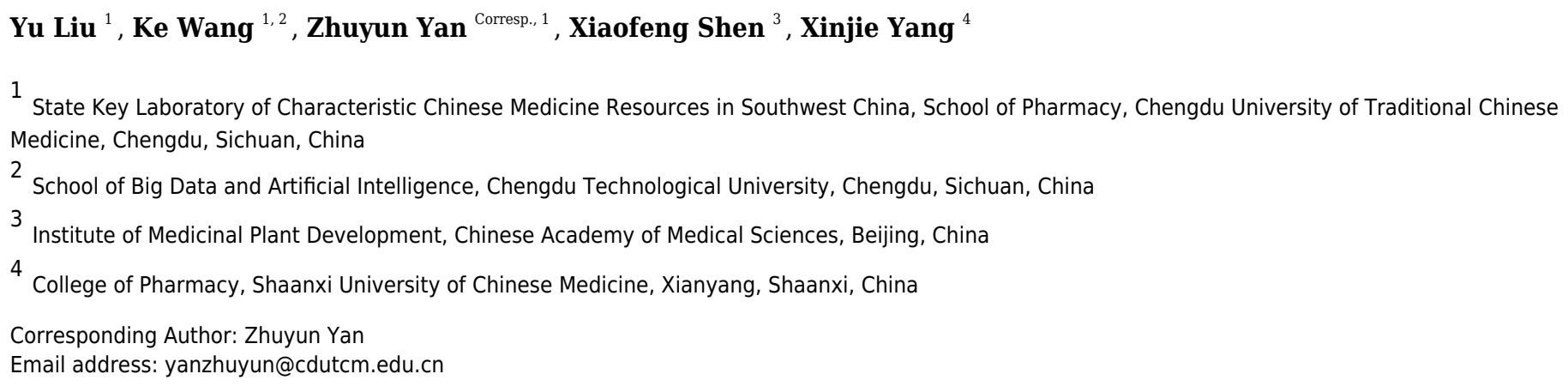

The roots of Salvia miltiorrhiza Bunge. are commonly used in the treatment of cardiovascular diseases, and tanshinones and salvianolic acids are its main active ingredients. However, the composition and content of active ingredients of S. miltiorrhiza planted in different regions of the soil environment are also quite different, which adds new difficulties to the large-scale and standardization of artificial cultivation. Therefore, in this study, we measured the active ingredients in the roots of $S$. miltiorrhiza and the contents of rhizosphere soil elements from 25 production areas in 8 provinces in China, and used the data to develop a prediction model based on BP (back propagation) neural network. The results showed that the active ingredients had different degrees of correlation with soil macronutrients and trace elements, the prediction model had the best performance (MSE $=0.0203,0.0164 ; R^{2}=0.93,0.94$ ). The artificial neural network model was shown to be a method that can be used to screen the suitable cultivation sites and proper fertilization. It can also be used to optimize the fertilizer application at specific sites. It also suggested that soil testing formula fertilization should be carried out for medicinal plants like $S$. miltiorrhiza, which is grown in multiple origins, rather than promoting the use of "special fertilizer" on a large scale. Therefore, the model is helpful for efficient, rational, and scientific guidance of fertilization management in the cultivation of S. miltiorrhiza. 


\section{Prediction of active ingredients in Salvia miltiorrhiza}

\section{Bunge. based on soil elements and artificial neural}

\section{3 network}

4 Yu Liu ${ }^{1}$, Ke Wang ${ }^{1,2}$, Zhuyun Yan ${ }^{1}$, Xiaofeng Shen ${ }^{3}$, Xinjie Yang ${ }^{4}$

5

$6{ }^{1}$ State Key Laboratory of Characteristic Chinese Medicine Resources in Southwest China,

7 School of Pharmacy, Chengdu University of Traditional Chinese Medicine, Chengdu, Sichuan, 8 China

$9{ }^{2}$ School of Big Data and Artificial Intelligence, Chengdu Technological University, Chengdu, 10 Sichuan, China

$11{ }^{3}$ Institute of Medicinal Plant Development, Chinese Academy of Medical Sciences, Beijing, 12 China

$13{ }^{4}$ College of Pharmacy, Shaanxi University of Chinese Medicine, Xianyang, Shaanxi, China 14

Corresponding Author:

Zhuyun Yan ${ }^{1}$

Chengdu University of Traditional Chinese Medicine, Chengdu, Sichuan, 611137, P.R. China

Email address: yanzhuyun@cdutcm.edu.cn

\section{Abstract}

The roots of Salvia miltiorrhiza Bunge. are commonly used in the treatment of cardiovascular diseases, and tanshinones and salvianolic acids are its main active ingredients. However, the composition and content of active ingredients of S. miltiorrhiza planted in different regions of the soil environment are also quite different, which adds new difficulties to the large-scale and standardization of artificial cultivation. Therefore, in this study, we measured the active ingredients in the roots of S. miltiorrhiza and the contents of rhizosphere soil elements from 25 production areas in 8 provinces in China, and used the data to develop a prediction model based on BP (back propagation) neural network. The results showed that the active ingredients had different degrees of correlation with soil macronutrients and trace elements, the prediction model had the best performance (MSE $=0.0203,0.0164 ; \mathrm{R}^{2}=0.93,0.94$ ). The artificial neural network model was shown to be a method that can be used to screen the suitable cultivation sites and proper fertilization. It can also be used to optimize the fertilizer application at specific sites. It 
33 also suggested that soil testing formula fertilization should be carried out for medicinal plants

34 like S. miltiorrhiza, which is grown in multiple origins, rather than promoting the use of "special 35 fertilizer" on a large scale. Therefore, the model is helpful for efficient, rational, and scientific 36 guidance of fertilization management in the cultivation of S. miltiorrhiza.

\section{Introduction}

Salvia miltiorrhiza Bunge. is a persistent herb of the genus Salvia of the mint family, Lamiaceae (Chinese Flora Commission of the Chinese Academy of Sciences 1974). In China, Japan, and the United States (Su et al. 2015), its dried roots are widely used as one of the most commonly used traditional medicinal herbs to treat cardiovascular diseases, especially angina pectoris and myocardial infarction (Wang et al. 2017). Researches have shown that diterpenoid quinones and hydrophilic phenolic acids are its principal bioactive components (Mei et al. 2019). As cardiovascular disease is a common and frequently-occurring disease (Jagannathan et al. 2019), the wild resources are unable to meet the ever-increasing market demand. Currently, 19 provinces in China, including Shandong, Henan, Sichuan, Hebei, Hubei, Jiangsu, Shanxi, and Shaanxi (Lu 2019), have been introduced and cultivated S. miltiorrhiza, but there are significant differences in the content of active ingredients in the same species planted in different regions (Huang et al. 2019; Yang et al. 2011b). Soil is the material basis for the survival of plants, and the abundance and deficiency of major and trace elements in soil affect plant growth and development and physiological and biochemical metabolism, which also affect the yield and the composition and content of active ingredients in medicinal plants (Zhang et al. 2018).

Fertilization is the primary way often used in agricultural production to improve soil nutrition, and proper fertilization is an important measure to ensure the yield and quality of medicinal plants. Therefore, analyzing the relationship between soil elements and active components of medicinal plants and establishing related models can provide data support and technical guidance for selecting suitable sites and fertilization management in the cultivation of S. miltiorrhiza.

Although the use of models based on the relationship between soil environment and crop quality or the quality of medicinal plants to predict suitable land, crop yield, and quality has received widespread attention, most studies have used regression analysis models to predict the relationship between the two ( $\mathrm{Li}$ et al. 2020). Regression analysis models are built on the assumption of some idealized linear relationship between predictor and response variables. However, environmental factors show very high intra- and inter-individual variability, which means that the biological responses of plants in response to the environment are uncertain and nonlinear in nature (Gago et al. 2010). Therefore, many biological interactions cannot be explained by simple stepwise algorithms or exact formulations, especially in complex or noisy data. Artificial neural network methods have proven effective in solving such problems in 
68 different disciplines (e.g., in vitro culture (Hesami \& Jones 2020), remote sensing studies (Wang 69 et al. 2019)). The artificial neural network methods can learn and create nonlinear and complex 70 relationships and can flexibly solve the complex problems of multiple interacting variables

71 (Bayat et al. 2019). It is one of the best techniques for extracting information from inaccurate and 72 nonlinear data (Caselli et al. 2009). Among them, BP neural network model is the most widely 73 used neural network model in the fields of economics, engineering, and botany (Armaghani et al. 74 2018; Chen et al. 2019). The BP neural network has been used to monitor crop growth and crop 75 yield prediction (Akbar et al. 2018; Wang et al. 2019). However, few reports on the application 76 of the BP neural network predict active ingredients of medicinal plants based on soil elements. 77 Therefore, in this study, we took S. miltiorrhiza as the research object, measured the active 78 ingredients in the roots of $S$. miltiorrhiza. and the contents of rhizosphere soil elements from 25 79 production areas in 8 provinces in China, analyzed the relationship between them, and 80 established a BP neural network model for the prediction of active ingredients using soil 81 elements as input values, which expands the application of artificial neural network methods in 82 the field of medicinal botany and also provides references for its application in other directions 83 in the field of medicinal plant cultivation, and verifies the feasibility of using artificial neural 84 network model to effectively improve the accuracy of the prediction of the content of active 85 ingredients of plants.

\section{Materials \& Methods}

\section{Sample collection and processing}

Roots and rhizosphere soil of S. miltiorrhiza (cultivation or wild) were collected from 25 producing areas in 8 provinces of China from mid-November to early December 2007 (Fig. 1, Table. S1). The samples were collected by the "S" parallel sampling and multi-point mixing method, each sample was collected at 20 to 25 points, and the rhizosphere soil was collected by the root shaking method, and finally, the samples for analysis were obtained by the quadratic method, of which $2 \mathrm{~kg} / \mathrm{sample}$ of rhizosphere soil and $5 \mathrm{~kg} /$ sample of medicinal parts were retained. After the samples were collected and quickly transported back to the laboratory, the herbs were processed routinely, and the soil samples were air-dried and prepared.

\section{Determination of inorganic elements}

The available potassium $(\mathrm{K})$, copper $(\mathrm{Cu})$, zinc $(\mathrm{Zn})$, and manganese $(\mathrm{Mn})$ were determined by atomic absorption spectrophotometry after ammonium bicarbonatediethylenetriaminepentaacetic acid (AB-DTPA) extraction (Lu 2000); the available nitrogen (N) was determined by alkaline diffusion method (Lin 2004); available phosphorus (P) was determined by sodium bicarbonate extraction-molybdenum antimony anti-colorimetric method

102 (Zhao et al. 2020). 
103 Determination of the content of active ingredients

104

105

106

107

108

109

110

111

112

113

114

115

116

117

118

119

120

121

122

123

124

125

126

127

128

129

130

131

132

133

134

135

136

137

138

A high-performance liquid chromatographic method was used to determine the contents of water-soluble components and lipid-soluble components (Yang et al. 2010; Yang et al. 2011a). The working parameters for the determination of water-soluble components was as follows: the column was a phenomenex Gemini $\mathrm{C}^{18}$ column $(250 \times 4.6 \mathrm{~mm}, 5 \mu \mathrm{m}$, Guangzhou Philomen Scientific Instruments Co., Ltd.); the mobile phases were water-acetonitrile-formic acid (90:10:0.4) (phase A) and acetonitrile (phase B), with gradient elution, phase A: 0 40min, 100\% 70\%; phase B: 0 40min, 0\% 30\%; the detection wavelength was $280 \mathrm{~nm}$; the flow rate was $1 \mathrm{~mL} / \mathrm{min}$; the column temperature was $25^{\circ} \mathrm{C}$; the injection volume was $20 \mu \mathrm{L}$. The working parameters for the determination of liposoluble components were as follows: the column was a Welchrom ${ }^{\mathrm{TM}} \mathrm{C}^{18}$ column (Analytial $4.6 \times 250 \mathrm{~mm}, 5 \mu \mathrm{m}$, Welch Corporation, USA); the mobile phases were methanol (A phase) and water (B phase), with gradient elution, A phase: 0 25 $\min , 67 \% \sim 67 \%$; 25 45 $\mathrm{min}, 67 \% \sim 90 \%$; B phase: 0-25 $\mathrm{min}, 33 \%-33 \%$; 25-45 $\mathrm{min}$, $33 \%-10 \%$. The detection wavelength was $270 \mathrm{~nm}$; the flow rate was $1 \mathrm{~mL} / \mathrm{min}$; the column temperature was $25^{\circ} \mathrm{C}$; the injection volume was $20 \mu \mathrm{L}$.

\section{BP (back propagation) neural network}

Bootstrap is a statistical inference method based on resampling and data simulation. Due to the nonlinear nature of small sample sizes and the difficulty of characterizing the overall distribution, the Bootstrap method can be applied to improve the estimation accuracy of the model (Wang et al. 2018). Therefore, the Bootstrap method has the potential to be widely applied to modeling estimations of small sample sizes. The steps are described as follows: (i) perform resampling to select a certain number (given) of samples and to allow repeat sampling; (ii) calculate the given statistics $\mathrm{T}$ based on the given samples; and (iii) repeat the above steps $\mathrm{N}$ times to gain $\mathrm{N}$ number of statistics $\mathrm{T}$ (Wang et al. 2018). The back propagation neural network proposed by Rumelhart in 1986 is a multi-layer feedforward network trained according to the error back propagation algorithm and is one of the most widely used neural network models (Rumelhart et al. 1986). The BP neural network model algorithm consists of two aspects: the forward propagation of the signal and the back propagation of the error. In other words, the actual output is calculated according to the direction from input to output. However, when the actual output contradicts the expected output, the back propagation of error is performed according to the direction from output to input, and the output error of each layer neuron is calculated layer by layer. Then, the weights and thresholds of each layer are adjusted according to the error gradient descent method to achieve the final output value that can be close to the expected value. The model-building process was described in detail as follows.

In this study, a BP neural network model was constructed using the neural fitting tool (nftool) in MATLAB 2019b mathematical software and trained with soil element parameters as 
139 input and the content of plant active ingredients as output. The input variables were as follows:

$140 \mathrm{Mn}, \mathrm{Cu}, \mathrm{Zn}, \mathrm{N}, \mathrm{P}$, and K; output variables: water-soluble components (danshensu, protocatechuic 141 aldehyde, caffeic acid, rosmarinic acid, salvianolic acid B, salvianolic acid A), and lipid-soluble 142 components (dihydrotanshinone I, cryptotanshinone, tanshinone I, and tanshinone IIA). In the 143 solution process, $70 \%$ of the samples $(n=18)$ were used to obtain samples using the bootstrap 144 method and build a BP neural network model. The remaining $30 \%$ of the samples $(n=8)$ were used to verify the model. First, to prevent the negative impact of different ranges of input variables on the model's efficiency, the input variables in the model were therefore normalized for the specified range (0-1) (Equation 1):

$F_{j}=\frac{X_{j}-X_{\min }}{X_{\max }-X_{\min }}$

148

149

150

151

152

153

$h=\sqrt{m+n}+a$

154

155

156

157

158

159

160

161

162

163

164

165

166

167

In the equation, $F_{j}, X_{j}, X_{\min }$ and $X_{\max }$ are the standardized value, original value, minimum value and maximum value of the input variable respectively.

Second, the number of hidden layers and the number of neurons in each hidden layer in a BP neural network impact the overall neural network structure. Currently, many empirical formulas are applied to determine the number of neurons in the hidden layer, and one of these formulas is as follows (Equation 2):

In the equation, $m(m=6)$ is the number of nodes in the input layer, $n(n=2)$ is the number of nodes in the output layer, and $a(1 \leq a \leq 10)$ is a constant.

According to equation (2), the number of nodes in the hidden layer was set as an integer between 4 and 12. Eventually, the number of neurons in the hidden layer was determined to be 8 by iterative trials. A neural network model with 6-8-2 structure was finally established (Fig. 2), in which the input layer consisted of 6 neurons corresponding to the 6 input variables, and the output layer had 2 neurons representing the content of active components in the model.

Third, another problem in establishing neural network models is the choice of network learning or training algorithms. Since the Levenberg-Marquardt algorithm minimizes the sum of the error function of the form (Equation 3), thus the Levenberg-Marquardt algorithm has the best performance (R-value) compared to other training algorithms (i.e., Bayesian regularization (BR) and scaled conjugate gradient (SCG)) (Mahmoudi \& Mahmoudi 2014). Therefore, the Levenberg-Marquardt algorithm will be used to train the network. The training epochs, learning rate, and minimum performance gradient were set as $1000,0.01$, and $1 \mathrm{e}^{-7}$.

$E=\frac{1}{2} \sum k\left(e_{k}\right)^{2}=\frac{1}{2}\|e\|^{2}$ 
168

169

170

171 (Yi et al. 2007).

$$
f(x)=\frac{1}{1+e^{x}}
$$

172 The output $y_{j}$ of the hidden layer neuron $\mathrm{j}$ is calculated by Equation 5:

$$
y_{j}=\varnothing\left(\sum_{i=1}^{n} W_{i j} X_{i}+\theta_{j}\right)
$$

173

In the equation, $\varnothing(x)$ represents the activation function of the hidden layer, $n$ is the number of neurons in the input layer, $X_{i}$ is the input of the input layer neuron $i, W_{i j}$ is the weight from the input layer neuron $i$ to the hidden layer neuron $j$, and $\theta_{j}$ is the threshold value of the hidden layer neuron $j$.

177 The output $z_{k}$ of the output layer neuron $k$ are calculated by Equation 6:

$$
z_{k}=f\left(\sum_{j=1}^{m} V_{j k} y_{j}+\alpha_{k}\right)
$$

178

179

180

181

182

183

184

185

186

187

188

189

190

191

In the equation, $f(x)$ represents the activation function of the output layer, and $m$ is the number of neurons in the hidden layer, $V_{j k}$ is the weight of the hidden layer neuron $j$ to the output layer neuron $k$, and $\alpha_{k}$ is the threshold value of the output layer neuron $k$.

The network weights adjustment is defined as follows (Equation 7) (Huang et al. 1996):

$\Delta \omega(t)=-\alpha \frac{\partial E}{\partial \omega(t)}+\beta \Delta \omega(t-1)$

Where $\alpha$ and $\beta$ are assumed constants, called the learning rate and momentum factor, respectively, $E$ is the error function (in which MSE was used), $\omega$ is the weight vector, and $t$ is the iteration number (epoch in MATLAB).

Finally, the steps of training can be summarized as follows: (i) applying the input vectors, (ii) calculating the output of the network and comparing it with the corresponding target vectors, (iii) feeding the difference (error) through the network, and (iv) changing the weights according to the algorithm, which tends to minimize the error. The vectors of the training set are applied sequentially. This process is repeated several times using the entire training set until the error is within acceptable criteria or until the output does not change significantly.

\section{Predictive performance evaluation}


192

193 194

$$
\begin{array}{r}
R^{2}=1-\frac{\sum_{\mathrm{i}=1}^{\mathrm{n}}\left(Y_{\mathrm{i}}-Y_{\mathrm{j}}\right)^{2}}{\sum_{\mathrm{i}=1}^{\mathrm{n}}\left(Y_{i}-\overline{Y_{i}}\right)^{2}} \\
M S E=\frac{1}{n} \sum_{i=1}^{n}\left(Y_{i}-\hat{Y}_{i}\right)^{2}
\end{array}
$$

195

To evaluate the predictive power of the model, the MSE (mean square error) and the $\mathrm{R}^{2}$ (coefficient of determination) were used to evaluate the overall performance of the model (Equation 8-9):

\section{In the equation, $Y_{i}$ is the} predicted data, $\bar{Y}_{i}$ is the average of the experimental data and $n$ is the number of experimental data.

\section{Data analysis}

In this study, correlation plots of the active ingredients of $S$. miltiorrhiza and rhizosphere soil elements were created using the "corrplot" package (version 0.9; cran.rproject.org/web/packages/corrplot/index.html) in R. The Pearson's correlation test was used to demonstrate the correlation between the active ingredients of $S$. miltiorrhiza and rhizosphere soil elements. $\mathrm{P}<0.05$ was considered to indicate a statistically significant difference. BP neural network model was created using the neural fitting tool (nftool) in MATLAB 2019b mathematical software (MathWorks Corporation, America). Scatter and regression plots were created using the "ggplot2" package (version 3.3.5; cran.rproject.org/web/packages/ggplot2/index.html) in R. The study used the kappa value to evaluate the multicollinearity of characteristic indicators by the "Kappa" package in R. The map plot was created using the "plyr" package (version 1.8.6; cran.rproject.org/web/packages/plyr/index.html), "maptools" package (version 1.1-2; cran.rproject.org/web/packages/maptools/index.html), and "ggplot2" package (version 3.3.5; cran.rproject.org/web/packages/ggplot2/index.html) in R.

\section{Results}

\section{Correlation analysis of active ingredients and rhizosphere soil elements}

In this study, the active ingredients and rhizosphere soil elements of S. miltiorrhiza from 25 producing areas in 8 provinces of China were determined, and the contents of 6 elements (Mn, $\mathrm{Cu}, \mathrm{Zn}, \mathrm{N}, \mathrm{P}, \mathrm{K}$ ) (Fig. S1) and 10 the kinds of active ingredients (danshensu (D1), protocatechuic aldehyde (D2), caffeic acid (D3), rosmarinic acid (D4), salvianolic acid B (D5), salvianolic acid A (D6), dihydrotanshinone I (D7)), cryptotanshinone (D8), tanshinone I (D9), tanshinone IIA 
220 (D10)) (Fig. S2). From the perspective of linear relationship, Figure 3 shows the correlation

221 between the content of different active ingredients and soil elements. Most active ingredients are 222 related to major and trace elements ( $r>0)$. In addition, the relationships between soil elements

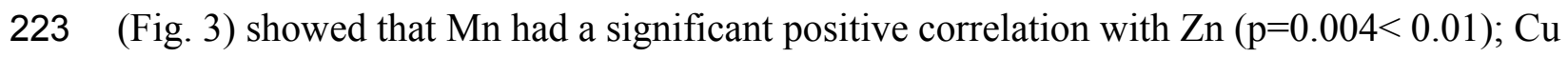

224 showed a weak correlation with $\mathrm{Zn}, \mathrm{K}(\mathrm{r}<0.4)$; N showed a weak correlation with $\mathrm{P}(\mathrm{r}<0.4)$; Zn

225 showed a weak correlation with $\mathrm{K}(\mathrm{r}<0.4)$. It is suggested that there were synergistic properties

226 in the utilization of elements by S. miltiorrhiza. Then, the study used the kappa value to evaluate

227 the multicollinearity of typical indicators. A k value below 100 was interpreted as low

228 multicollinearity, and a k value exceeding 1000 indicates high multicollinearity (Ma et al. 2021).

229 The kappa value $(\mathrm{k}=480.153)$ was exceeded 100. These results indicated multicollinearity among

230 fundamental soil indicators, and linear regression cannot be performed directly. From the

231 perspective of a nonlinear relationship, Fig. 4 and Fig. 5 showed the scatter and regression plots

232 between the active ingredients of S. miltiorrhiza and the soil elements. The results showed that

233 there was not a simple increase or decrease between the active ingredients of S. miltiorrhiza and

234 soil elements, but an inevitable fluctuation would follow, which means that as the content of soil

235 elements increases, the content of active ingredients of $S$. miltiorrhiza will increase and decrease.

236 These results indicated that the active ingredients of $S$. miltiorrhiza had a nonlinear relationship

237 with multiple soil elements, and a particular element index cannot be used to express the

238 effective ingredients of S. miltiorrhiza. The artificial neural network methods can learn and

239 create nonlinear and complex relationships and can flexibly solve the complex problems of

240 multiple interacting variables (Bayat et al. 2019). It is one of the best techniques for extracting

241 information from inaccurate and nonlinear data (Caselli et al. 2009). Therefore, in this study, a

242 BP neural network model with soil elements as input values was established to explore the

243 relationship between soil elements and the active ingredients for predicting the active

244 ingredients.

\section{Predictive performance of BP neural network model}

246 In this study, a BP neural network model was constructed using the neural fitting tool

247 (nftool) in MATLAB 2019b mathematical software and trained with soil element parameters as

248 input and the content of plant active ingredients as output. The input variables were as follows:

$249 \mathrm{Mn}, \mathrm{Cu}, \mathrm{Zn}, \mathrm{N}, \mathrm{P}$, and K; output variables: water-soluble components (danshensu, protocatechuic

250 aldehyde, caffeic acid, rosmarinic acid, salvianolic acid B, salvianolic acid A), and lipid-soluble

251 components (dihydrotanshinone I, cryptotanshinone, tanshinone I, and tanshinone IIA). Through

252 repeated training, a neural network model with 6-8-2 structure was finally established (Fig. 2). In

253 order to evaluate the predictive ability of the established BP neural network model, the MSE

254 (mean square error) and the coefficient of determination $\left(\mathrm{R}^{2}\right)$ were used to evaluate the overall

255 performance of the model. Ideally. The closer the MSE value is to zero, the closer the $\mathrm{R}^{2}$ value is 
256 to 1 , which indicates that the average training and testing performance is appropriate. The BP 257 model showed fast training and high simulation accuracy in model testing (Figure 6A). The 258 relationship between the predicted and measured active ingredients content was favorable, 259 thereby indicating that this model has a basic consistency and a high degree of simulation. A 260 scatter diagram was constructed between the predicted and real values of the inversion model. 261 The coefficient of determination $\mathrm{R}^{2}$ between the predicted and real values of the inversion model 262 was 0.93 , the linear regression line between the measured and predicted values were close to 1 (i.e., linear), and the MSE (MSE=0.0203) was low. Therefore, the predictive ability of the model

264

265

266

267

268

269

270

271

272

273

274

275

276

277

278

279

280

281

282

283

284

285

286

287 was relatively high and showed strong nonlinear fitting ability, indicating that the soil elemental content could be used for accurate inversion of the active ingredient content.

From the results obtained, the empirical equation based on the Levenberg-Marquardt algorithm for predicting active ingredient content in normalized form is Equation 10:

$(D 1, D 2)=\sum_{j=1}^{3}\left[\operatorname{purelin}\left\{\sum_{i=1}^{8} \sum_{j=1}^{6} \log \operatorname{sig}\left[\left(M n j_{1}+C u j_{2}+Z n j_{3}+N j_{4}+P j_{5}+K J_{6}\right)+b_{i}\right]\right\} \times L w_{i, j}+b_{k_{i}}\right]$

The transfer function "purelin" correlated the linear relationship between the input and output variables, while "logsig" calculated the layer's output from the network input. The variables $j_{1}, j_{2}, j_{3}, j_{4}, j_{5}$, and $j_{6}$ were input weights from the input layer to the hidden layer. Also, $L w_{1}, L w_{2}$ were hidden layer weights from the hidden layer to the output layer. The variables $b_{i}$ and $b_{k}$ were biases connected to the hidden layer neurons and output layer neurons, respectively. The values for these weights and biases for the model were in Table 1.

To further evaluate the model's generalizability, the developed neural network model was tested using 41 new datasets. The new datasets were derived from the results of Zhang's study (Zhang et al. 2018b). The correlation coefficient, mean square error, was used to evaluate the generalization ability of the model. A scatter diagram (Fig. 6B) was constructed between the predicted and real values of the inversion model. The coefficient of determination $\mathrm{R} 2$ between the predicted and real values of the inversion model was 0.94 , the linear regression line between the measured and predicted values were close to 1:1 (i.e., linear), and the MSE (MSE=0.0164) was low. Therefore, the predictive ability of the model was relatively high. Our results indicate that the BP neural network model based on the content of soil elemental can be a powerful tool for predicting the content of active ingredients of $S$. miltiorrhiza.

\section{Discussion}

Soil is the material basis for the survival of plants. The large and trace elements in the soil can provide nutrients for plants, provide good growth and metabolic status, and enhance their resistance to adversity (Alam \& Naik 2009; Zhang et al. 2018). These are the basis for the 
288

289

290

291

292

293

294

295

296

297

298

299

300

301

302

303

304

305

306

307

308

309

310

311

312

313

314

315

316

317

318

319

320

321

322

323

production of secondary metabolites of the plant. Isopentenyl diphosphate (IPP) and its isomer dimethylallyl diphosphate (DMAPP) are the precursors of all terpenoids (Ma et al. 2015). The synthesis of both requires $\mathrm{P}$. The phenylpropanoid pathway and the tyrosine-derived pathway are the main pathways for the biosynthesis of salvianolic acids (Ma et al. 2015). The synthesis of phenylalanine, the starting point of the phenylpropane pathway, requires the participation of $\mathrm{N}$. $\mathrm{K}$ not only regulates plant water metabolism but also acts as an activator of enzymes involved in respiration and photosynthesis (e.g., glutathione synthase) (Liu 2009). Trace elements ( $\mathrm{Mn}, \mathrm{Cu}$, $\mathrm{Zn}$ ) are involved in the biosynthesis of plant active ingredients as cofactors, constituent elements, and activators of some enzymes (Guo et al. 2005). Therefore, the biosynthesis of the active ingredients of $S$. miltiorrhiza cannot be achieved without the participation of soil elements. In this study, the results of correlation analysis between soil elements and active ingredients based on the harvesting period of the herb showed that the active ingredients of S. miltiorrhiza interacted with several soil elements and showed a nonlinear relationship, which may be different from the results of fertilization experiments, for example, Han and Liang (2005) reported that phosphorus fertilization showed a positive effect on the accumulation of danshensu and tanshinone IIA, but this effect was not observed for the effective phosphorus content in this study. Combined with the Mao's research results (Mao et al. 2009), it is believed that it is a matter of elemental availability or antagonism in the utilization between elements. Moreover, there were different levels of correlation between the affective states of the elements, suggesting a synergistic or antagonistic effect on elemental uptake by $S$. miltiorrhiza. The results of our study further suggest that soil testing formula fertilization should be implemented for medicinal plants like $S$. miltiorrhiza, which is grown in multiple origins, rather than promoting the use of "special fertilizer" on a large scale. The relationship between fertilizer requirements of medicinal plants and soil supply should be coordinated. The required nutrients should be supplemented in a targeted manner to achieve a balanced supply of nutrients and guide the fertilization management of medicinal plants in an efficient, rational, and rational scientific manner. Therefore, it is imperative to establish a method that can be used for screening suitable cultivation sites and rational fertilization of medicinal plants.

Here, we presented and validated the application of artificial neural networks in predicting the active ingredients of medicinal plants. Artificial neural networks have received more attention in various applications due to their sensitivity, accuracy, non-destructive nature, and rapidity (Wu et al. 2012). Many researchers have used artificial neural networks to monitor crop growth and crop yield prediction (Akbar et al. 2018; Wang et al. 2019; Yang et al. 2018), but there are few reports on the use of artificial neural networks to predict active ingredients of medicinal plants based on soil elements. Our results showed that artificial neural network models provided more appropriate predictions of the content of protocatechuic aldehydes, salvianolic

Peer] reviewing PDF | (2021:07:63562:2:0:NEW 6 Dec 2021) 
324 acids, tanshinones, and dihydrotanshinone I using the site's soil elemental characteristics 325 conditions. We recorded minimal differences between the predicted and observed data (MSE $\left.326=0.0203,0.0164 ; \mathrm{R}^{2}=0.93,0.94\right)$, and the excellent agreement between the results support the 327 high efficiency of artificial neural networks while demonstrating that the use of mathematical 328 models (e.g., artificial neural networks) to predict the active ingredient content in medicinal 329 plants can reduce the time and cost required for analytical methods. Artificial neural network 330 models offer significant advantages over traditional mathematical models by using nonlinear

331

332

333

334 335 336 337 338 339 340

341

342 network connections and allowing analysis that explores the efficacy of all input variables simultaneously, thereby improving the accuracy of results (Alam \& Naik 2009; Tušek et al. 2018). However, the capability of artificial neural network models is usually also limited by drawbacks such as slow learning speed, overfitting, and local minima, which suggests that building some hybrid neural network models to reduce their drawbacks and improve their performance is an essential direction for future research.

Many medicinal plants have greater medicinal productivity in their original habitat than in cultivated areas. Soil nutrient characteristics similar to the original habitat must be best suited for the production of active metabolites (Alam \& Naik 2009). Based on the results of this study, an artificial neural network model can be used to screen suitable cultivation sites for medicinal plants and simulate soil conditions similar to the original soil conditions through soil management, thus increasing the yield of active ingredients. The specific measures are as follows: firstly, measuring the key nutrient factors of the soil, such as massive elements and trace elements; and finally balancing fertilization, such as targeted supplementation of required nutrients during plant growth and harvest, to achieve a balanced supply of nutrients and to efficiently, rationally and scientifically guide the fertilization management of medicinal plants. However, the soil matrix is a complex organic ecosystem, while the plants' secondary metabolites themselves are a complex physiological process, so the effect of soil on plant active ingredients is far more complex than what is involved in the current study. So future work could also extend this modeling process by using a more inclusive range of biotic and abiotic variables to obtain more accurate estimates.

\section{Conclusions}

In this study, an artificial neural network model for predicting the active ingredients of $S$. miltiorrhiza using rhizosphere soil elements as input values were developed to localize the effects of these factors on the active ingredients. This expands the application of artificial neural network methods in medicinal botany and provides a reference for other directions in the field of medicinal plant cultivation. The results show that, in combination with soil data, we can use artificial neural network models to successfully predict the content of protocatechuic aldehyde, 
359

360

361

362

363

364

365

366

367

368

369

370

371

372

373

374

375

376

377

378

379

380

381

382

383

384

385

386

387

388

389

390

391

392

393

394

395

396

salvianolic acids, tanshinones, and dihydrotanshinone I. This further validates the feasibility that artificial neural network models can effectively improve the accuracy of the prediction of the active ingredient content of medicinal plants. In addition, the model can provide broader applicability for ranch managers, manufacturers, and producers of medicinal plants to screen suitable sites for medicinal plant cultivation in a robust and reproducible manner. It can also optimize the fertilizer application at specific sites and guide the fertilization management of medicinal plants in an efficient, rational, and rational scientific manner.

\section{References}

Akbar A, Kuanar A, Patnaik J, Mishra A, and Nayak S. 2018. Application of artificial neural network modeling for optimization and prediction of essential oil yield in turmeric (Curcuma longa L.). Computers and Electronics in Agriculture 148:160-178. https://doi.org/10.1016/j.compag.2018.03.002

Alam MA, and Naik PK. 2009. Impact of soil nutrients and environmental factors on podophyllotoxin content among 28 Podophyllum hexandrum populations of northwestern Himalayan region using linear and nonlinear approaches. Communications in soil science and plant analysis 40:2485-2504. https://doi.org/10.1080/00103620903111368

Armaghani DJ, Hasanipanah M, Mahdiyar A, Abd Majid MZ, Amnieh HB, and Tahir MM. 2018. Airblast prediction through a hybrid genetic algorithm-ANN model. Neural Computing and Applications 29:619-629. https://doi.org/10.1007/s00521-016-2598-8

Bayat M, Ghorbanpour M, Zare R, Jaafari A, and Pham BT. 2019. Application of artificial neural networks for predicting tree survival and mortality in the Hyrcanian forest of Iran. Computers and Electronics in Agriculture 164:104929. https://doi.org/10.1016/j.compag.2019.104929

Caselli M, Trizio L, De Gennaro G, and lelpo P. 2009. A simple feedforward neural network for the PM 10 forecasting: Comparison with a radial basis function network and a multivariate linear regression model. Water, Air, and Soil Pollution 201:365-377. https://doi.org/10.1007/s11270-008-9950-2

Chen RP, Zhang P, Kang X, Zhong ZQ, Liu Y, and Wu HN. 2019. Prediction of maximum surface settlement caused by earth pressure balance (EPB) shield tunneling with ANN methods. Soils and Foundations 59:284-295. https://doi.org/10.1016/j.sandf.2018.11.005

Chinese Flora Commission of the Chinese Academy of Sciences. 1974. Flora of China: Volume 66. Beijing: Science Press.

Gago J, Martínez-Núñez L, Landín M, and Gallego P. 2010. Artificial neural networks as an alternative to the traditional statistical methodology in plant research. Journal of plant physiology 167:23-27. https://doi.org/10.1016/j.jplph.2009.07.007

Guo XH, Gao WY, Chen HX, and Huang LQ. 2005. Effects of mineral cations on the accumulation of tanshinone II $A$ and protocatechuic aldehyde in the adventitious root culture of Salvia niltiorrhiza. China Journal of Chinese Materia Medica 30:885-888.

Peer] reviewing PDF | (2021:07:63562:2:0:NEW 6 Dec 2021) 
397

398

399

400

401

402

403

404

405

406

407

408

409

410

411

412

413

414

415

416

417

418

419

420

421

422

423

424

425

426

427

428

429

430

431

432

433

434

435

436

Han JP, and Liang ZS. 2005. Regulation of Salvia miltiorrhizae growth and danshensu and tanshion II A accumulation under nitrogen and phosphorus. Chinese Traditional and Herbal Drugs:756-759.

Hesami M, and Jones AMP. 2020. Application of artificial intelligence models and optimization algorithms in plant cell and tissue culture. Applied Microbiology and Biotechnology 104:9449-9485. https://doi.org/10.1007/s00253-020-10888-2

Huang XL, Xiong L, Wang YM, Xu F, Liang YQ, Wang XP, and Wu HM. 2019. Evaluation of quality of Salvia miltiorrhiza Bunge from different provenances by HPLC-DAD fingerprint combined with Chemometrics Method. IOP Conference Series: Earth and Environmental Science: IOP Publishing. p 012163.

Huang ZH, Shimeld J, Williamson M, and Katsube J. 1996. Permeability prediction with artificial neural network modeling in the Venture gas field, offshore eastern Canada. Geophysics 61:422-436. https://doi.org/10.1190/1.1443970

Jagannathan R, Patel SA, Ali MK, and Narayan KV. 2019. Global updates on cardiovascular disease mortality trends and attribution of traditional risk factors. Current diabetes reports 19:1-12. https://doi.org/10.1007/s11892-019-1161-2

Li ZT, Liu DX, Zhan LJ, and Li LH. 2020. Mineral elements and active ingredients in root of wild Paeonia lactiflora growing at Duolun County, Inner Mongolia. Biological trace element research 193:548-554. https://doi.org/10.1007/s12011-019-01725-3

Lin DY. 2004. Soil Science Experiment Guidance. Beijing: China Forestry Publishing House.

Liu YH. 2009. The role of nitrogen, phosphorus and potassium elements and the symptoms of different levels. The Journal of Hebei Forestry Science and Technology 000:57.

Lu RK. 2000. Soil agricultural chemical analysis method. Beijing: China Agricultural Science and Technology Press.

Lu SF. 2019. Salvia miltiorrhiza: An Economically and Academically Important Medicinal Plant. The Salvia miltiorrhiza Genome: Springer, 1-15. https://doi.org/10.1007/978-3-03024716-4_1

Ma BQ, Hauer RJ, Xu CY, and Li WJ. 2021. Visualizing evaluation model of human perceptions and characteristic indicators of landscape visual quality in urban green spaces by using nomograms. Urban Forestry \& Urban Greening 65:127314. https://doi.org/10.1016/j.ufug.2021.127314

Ma XH, Ma Y, Tang JF, He YL, Liu YC, Ma XJ, Shen Y, Cui GH, Lin HX, and Rong QX. 2015. The biosynthetic pathways of tanshinones and phenolic acids in Salvia miltiorrhiza. Molecules 20:16235-16254. https://doi.org/10.3390/molecules200916235

Mahmoudi S, and Mahmoudi A. 2014. Water saturation and porosity prediction using backpropagation artificial neural network (BPANN) from well log data. Journal of Engineering and Technology (JET) 5:1-8.

Mao Y, Yuan Y, He XR, and Cui GH. 2009. The Effects of Different Element on the Growth of Salvia Miltiorrhiza Hairy Roots and the Accumulate of Tanshinones in Salvia Miltiorrhiza. Chinese Journal of Experimental Traditional Medical Formulae 15:6-8. 
437 Mei XD, Cao YF, Che YY, LI J, Shang ZP, Zhao WJ, Qiao YJ, and Zhang JY. 2019. Danshen: a

438

439

440

441

442

443

444

445

446

447

448

449

450

451

452

453

454

455

456

457

458

459

460

461

462

463

464

465

466

467

468

469

470

471

472

473

474

475

476

477 phytochemical and pharmacological overview. Chinese journal of natural medicines 17:59-80. https://doi.org/10.1016/S1875-5364(19)30010-X

Rumelhart DE, Hinton GE, and Williams RJ. 1986. Learning representations by backpropagating errors. nature 323:533-536. https://doi.org/10.1038/323533a0

Su CY, Ming QL, Rahman K, Han T, and Qin LP. 2015. Salvia miltiorrhiza: Traditional medicinal uses, chemistry, and pharmacology. Chinese journal of natural medicines 13:163-182. https://doi.org/10.1016/S1875-5364(15)30002-9

Tušek AJ, Benković M, Valinger D, Jurina T, Belščak-Cvitanović A, and Kljusurić JG. 2018. Optimizing bioactive compounds extraction from different medicinal plants and prediction through nonlinear and linear models. Industrial Crops and Products 126:449-458. https://doi.org/10.1016/j.indcrop.2018.10.040

Tang ZY, and Fishwick PA. 1993. Feedforward neural nets as models for time series forecasting. ORSA journal on computing 5:374-385. https://doi.org/10.1287/ijoc.5.4.374

Tušek AJ, Benković M, Valinger D, Jurina T, Belščak-Cvitanović A, and Kljusurić JG. 2018. Optimizing bioactive compounds extraction from different medicinal plants and prediction through nonlinear and linear models. Industrial Crops and Products 126:449-458. https://doi.org/10.1016/j.indcrop.2018.10.040

Wang L, Wang PX, Liang SL, Qi X, Li L, and Xu LX. 2019. Monitoring maize growth conditions by training a BP neural network with remotely sensed vegetation temperature condition index and leaf area index. Computers and Electronics in Agriculture 160:82-90. https://doi.org/10.1016/j.compag.2019.03.017

Wang LL, Ma RF, Liu CY, Liu HX, Zhu RY, Guo SZ, Tang MK, Li Y, Niu JZ, and Fu M. 2017. Salvia miltiorrhiza: a potential red light to the development of cardiovascular diseases. Current Pharmaceutical Design 23:1077-1097. https://doi.org/10.2174/13816128226666161010105242

Wang XP, Zhang F, Ding JL, Latif A, and Johnson VC. 2018. Estimation of soil salt content (SSC) in the Ebinur Lake Wetland National Nature Reserve (ELWNNR), Northwest China, based on a Bootstrap-BP neural network model and optimal spectral indices. Science of the Total Environment 615:918-930. https://doi.org/10.1016/j.scitotenv.2017.10.025

Wu B, Liu Y, and Lu JQ. 2012. New results on global exponential stability for impulsive cellular neural networks with any bounded time-varying delays. Mathematical and Computer Modelling 55:837-843. https://doi.org/10.1016/j.mcm.2011.09.009

Yang SX, Feng QS, Liang TG, Liu BK, Zhang WJ, and Xie HJ. 2018. Modeling grassland above-ground biomass based on artificial neural network and remote sensing in the Three-River Headwaters Region. Remote Sensing of Environment 204:448-455. https://doi.org/10.1016/j.rse.2017.10.011

Yang XJ, Wan DG, Lin GB, Zhang Q, Wang JY, and Yan ZY. 2010. Analysis on geographical distribution of liposoluble con-stituents in Salvia miltiorrhiza. Chinese Traditional and Herbal Drugs 41:809-812. 
478 Yang XJ, Wan DG, Liu M, Lin GB, Wang JY, and Yan ZY. 2011a. Analysis on Geographical 479 Distribution of Hydrophilic Components in Radix Salviae Miltiorrhizae. Natural Product $480 \quad$ Research and Development 23:98-102.

481 Yang XJ, Wan DJ, Lin GB, Guo XH, Liu T, and Yan ZY. 2011b. The quality analysis of $482 \quad$ introduced Salvia miltiorrhiza from different provenances. Journal of Chengdu Medical $483 \quad$ College 006:291-295.

484 Zhang F, Yu X, Ma Y, Wang L, and Zhang YQ. 2018. Effects of Soil factors on the growth of $485 \quad$ Lonicera japonica Thunb. and the quality of honeysuckle flower. Lishizhen Medicine and Materia Medica Research 29:187-189. https://doi.org/10.3969/j.issn.10080805.2018.07.063

Zhang XD, Yu YG, Yang DF, Qi ZC, Liu RZ, Deng FT, Cai ZX, Li Y, Sun YF, and Liang ZS. 2018 b. Chemotaxonomic variation in secondary metabolites contents and their correlation between environmental factors in Salvia miltiorrhiza Bunge from natural habitat of China. Industrial Crops and Products 113:335-347. https://doi.org/10.1016/j.indcrop.2018.01.043

Zhao L, Li JN, Tie XW, and Li XY. 2020. Determination of soil available phosphorus based on sodium bicarbonate extrac-tion-molybdenum antimony spectrophotometry. Technology and Economic Guide 28:86. 
Figure 1

Part of China map indicating the location of the sample sites.

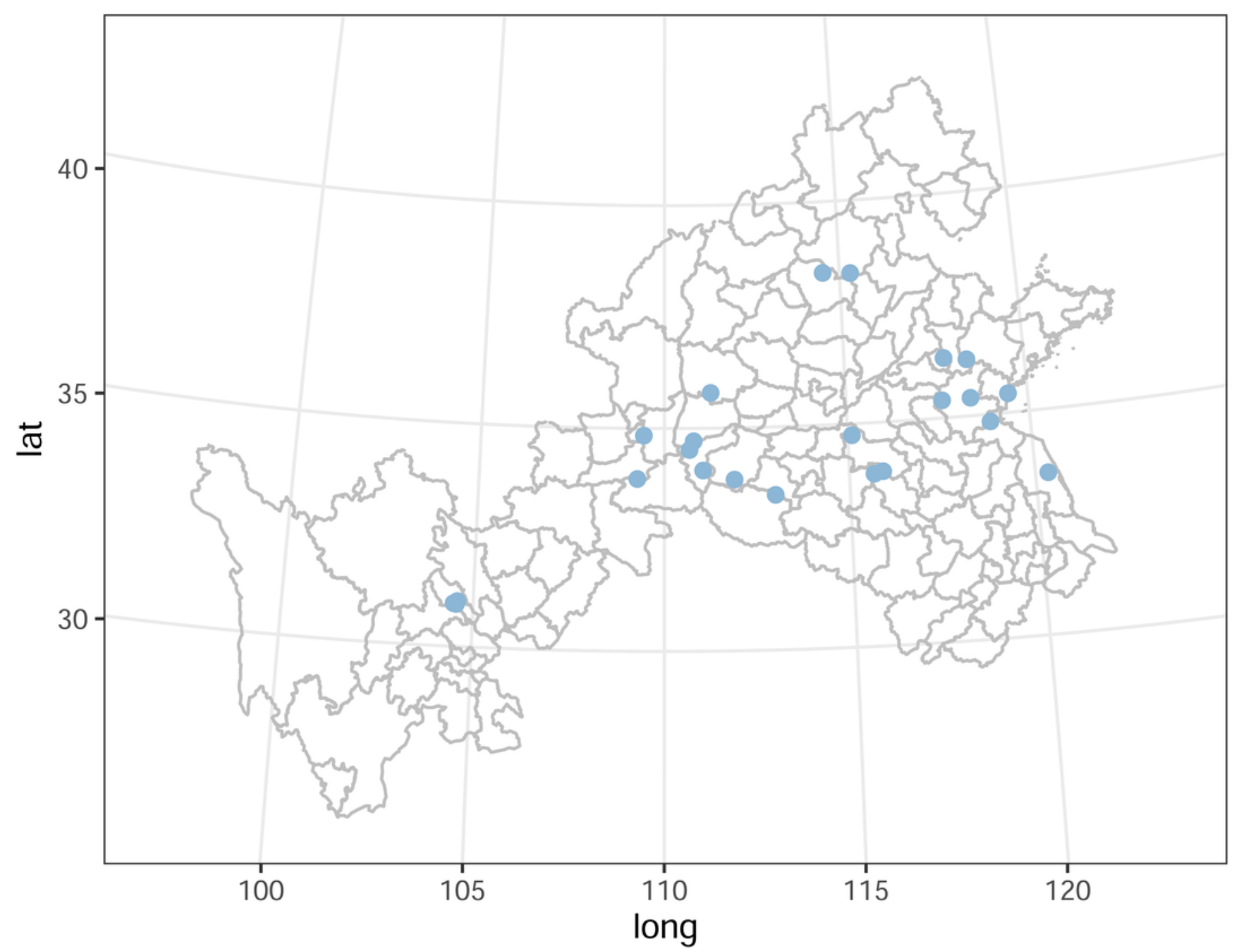


Figure 2

Structure of the back propagation neural network.

D1, D2 represents water-soluble components and fat-soluble components.

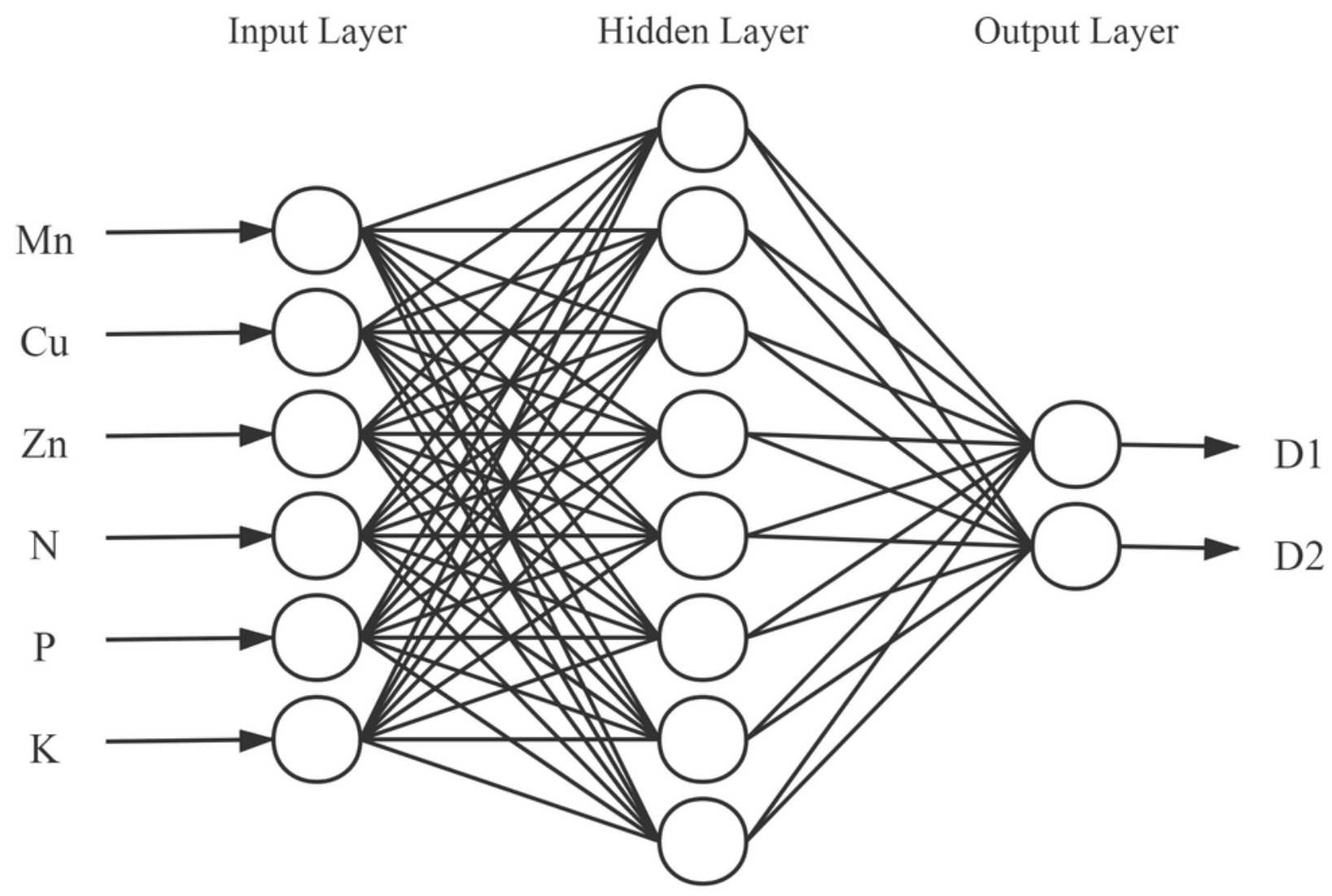




\section{Figure 3}

Correlation diagram between active ingredients and soil elements.

D1-D10 represents danshensu, protocatechuic aldehyde, caffeic acid, rosmarinic acid, dihydrotanshinone I, cryptotanshinone, tanshinone I, tanshinone IIA, salvianolic acid B, salvianolic acid A. Blue indicates positive correlation, red indicates negative correlation. The numbers and the size of the circle represented the correlation coefficients. The larger the area and number, the greater the correlation. 


\begin{tabular}{|c|c|c|c|c|c|c|c|c|c|c|c|c|c|c|c|}
\hline $\mathrm{Mn}$ & O & & 0 & 0 & P & O & 。 & O & & O & O & & (1) & 0 & 0 \\
\hline & $\mathrm{Cu}$ & & & (1) & 0 & 0 & (1) & & & & 0 & O & (1) & - & 0 \\
\hline 0.54 & 0.36 & $\mathrm{Zn}$ & & (1) & 0 & (1) & 0 & 0 & ○ & 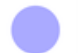 & ○ & (1) & & (1) & \\
\hline-0.21 & & & $\mathrm{~N}$ & 0 & & (1) & 0 & o & & (1) & 0 & & & & \\
\hline-0.24 & & & 0.34 & P & & 0 & & 0 & 0 & & - & () & & & \\
\hline 0.21 & 0.39 & 0.34 & & -0.16 & K & 0 & 。 & , & 0 & D & & 0 & & & \\
\hline & & & & 0.32 & & D1 & & & & & 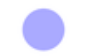 & & & & \\
\hline & & & & 0.42 & & 0.65 & D2 & & 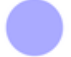 & & & 0 & & & \\
\hline & & -0.24 & & 0.14 & -0.36 & 0.33 & 0.47 & D3 & 0 & & 0 & & • & - & 。 \\
\hline & & & & 0.16 & & 0.73 & 0.39 & 0.41 & D4 & & 0 & (c) & (1) & ○ & ) \\
\hline & & 0.28 & & 0.31 & & 0.59 & 0.40 & 0.26 & 0.56 & D5 & 0 & & • & 0 & 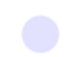 \\
\hline & -0.29 & & 0.25 & 0.02 & & 0.20 & 0.33 & 0.26 & 0.31 & 0.34 & D6 & (1) & 0 & & \\
\hline & & & & -0.37 & -0.21 & & -0.28 & & & & & D7 & & & \\
\hline & & 0.27 & & -0.35 & & & -0.28 & & & & & 0.81 & D8 & & \\
\hline & & & & -0.13 & & & -0.29 & & & & & 0.73 & 0.81 & D9 & \\
\hline & & 0.31 & & -0.24 & & & -0.39 & & & & & 0.68 & 0.92 & 0.82 & D10 \\
\hline
\end{tabular}


Figure 4

The scatter and regression plots between the water-soluble components of $S$. miltiorrhiza and the soil elements.

D1-D6 represents danshensu, protocatechuic aldehyde, caffeic acid, rosmarinic acid, salvianolic acid B, salvianolic acid A. 

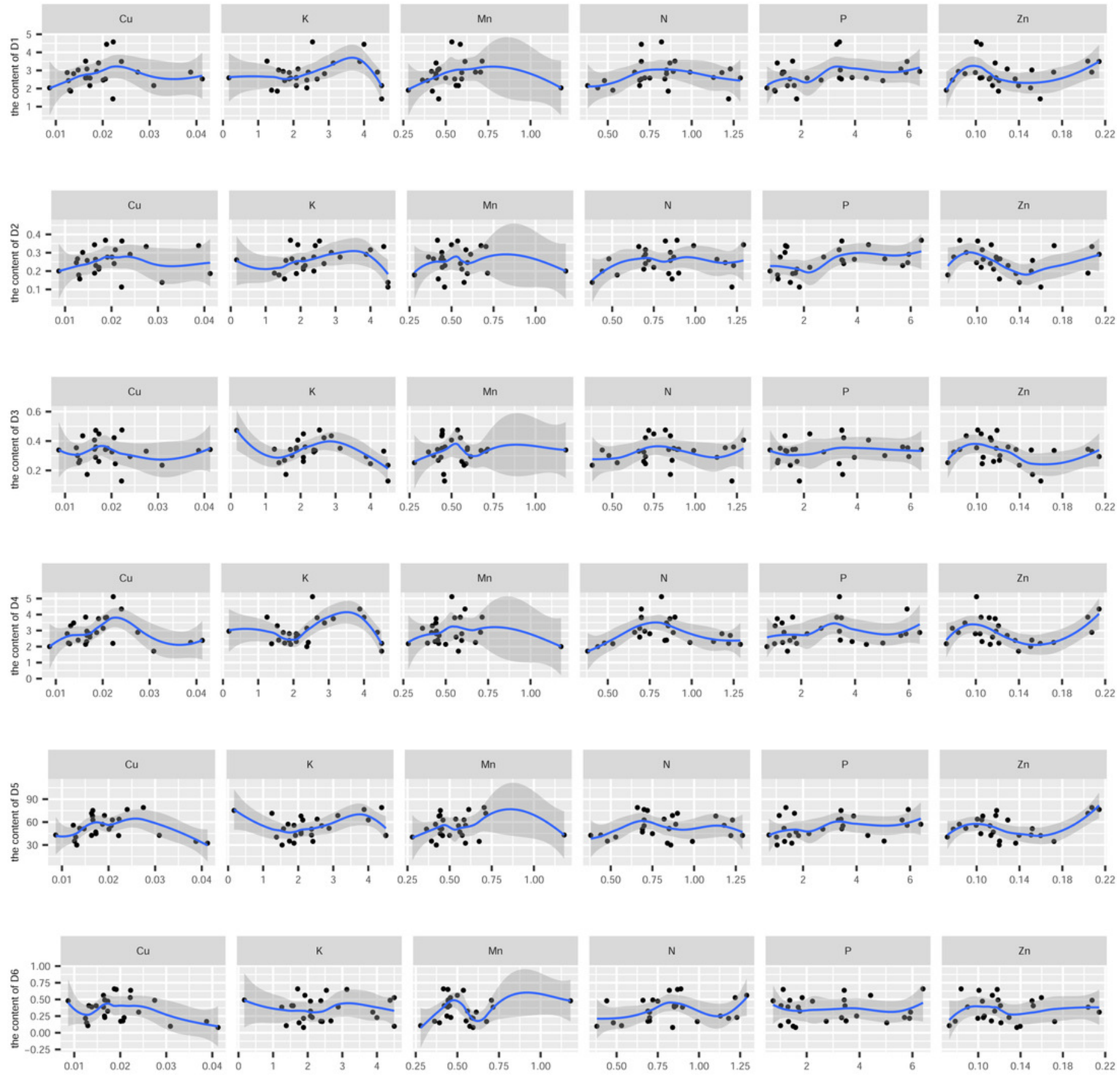


\section{Figure 5}

The scatter and regression plots between the lipid-soluble components of S. miltiorrhiza and the soil elements.

D7-D10 represents dihydrotanshinone I, cryptotanshinone, tanshinone I, tanshinone IIA.
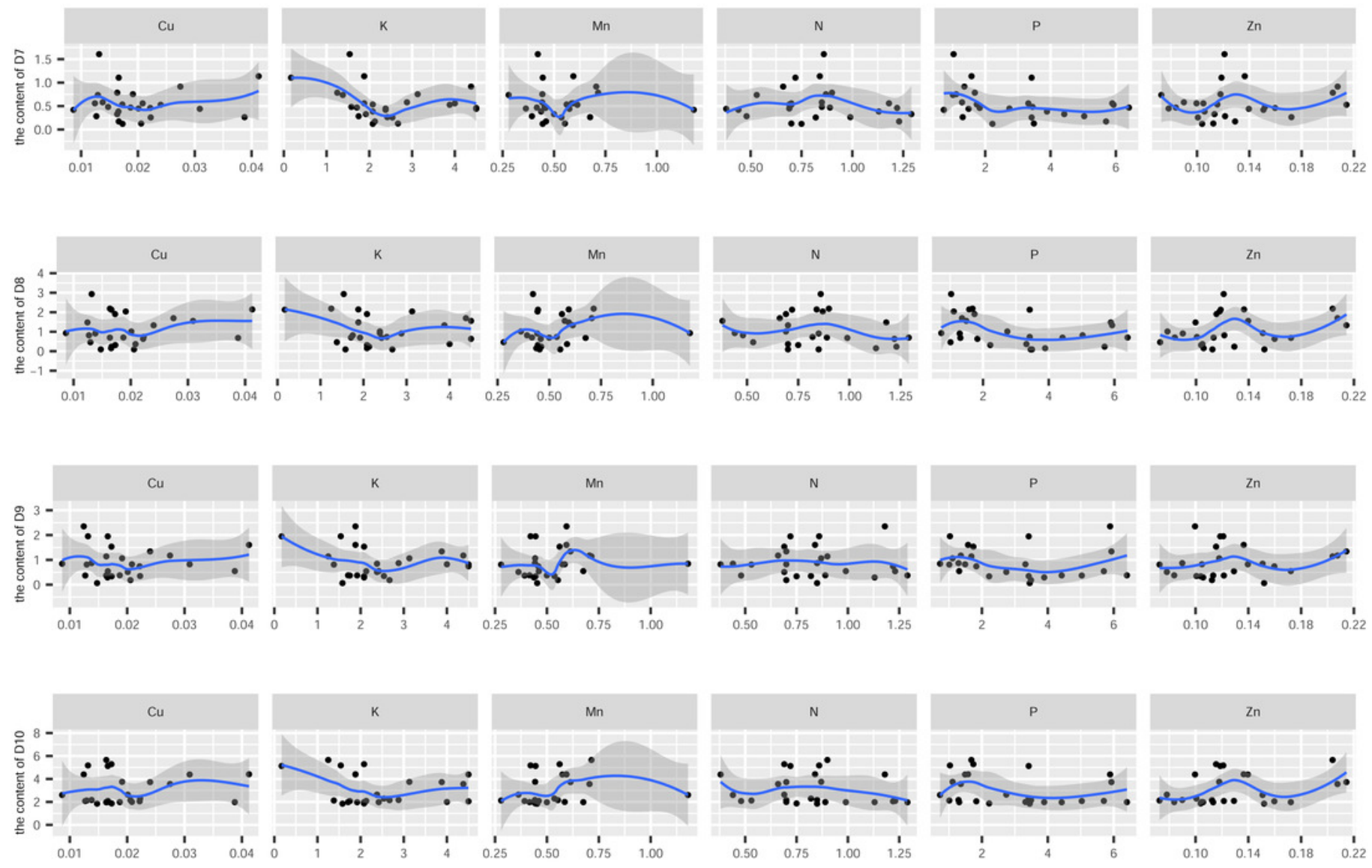
Figure 6

Scatter plots of the BP neural network outputs versus targets values.

(A) Scatter plot of outputs versus targets values of dataset $(n=26)$; (B) Scatter plot of outputs versus targets values of new dataset $(n=41)$.
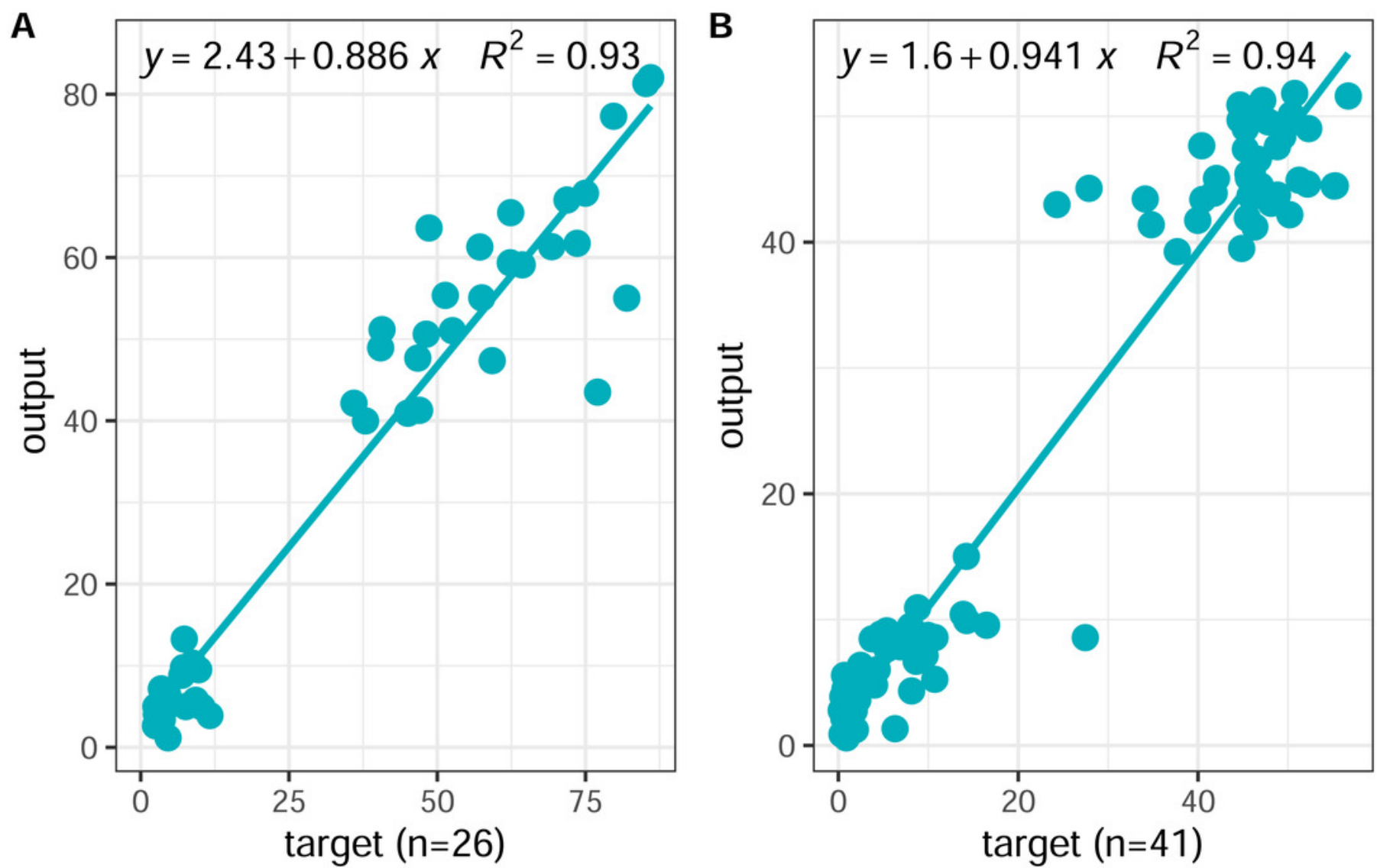


\section{Table $\mathbf{1}$ (on next page)}

Weights and biases of the model 
1 Table 1 Weights and biases of the model

\begin{tabular}{|c|c|c|c|c|c|c|c|c|c|c|}
\hline \multirow[t]{2}{*}{$i$} & \multicolumn{6}{|c|}{ Input weights } & \multirow{2}{*}{$\begin{array}{l}\text { Output layer } \\
\text { biases } \\
b_{I}\end{array}$} & \multicolumn{2}{|c|}{ Hidden layer weights } & \multirow{2}{*}{$\begin{array}{l}\text { Output layer } \\
\text { biases } \\
b_{k}\end{array}$} \\
\hline & $j_{l}(\mathrm{Mn})$ & $j_{2}(\mathrm{Cu})$ & $j_{3}(\mathrm{Zn})$ & $j_{4}(\mathrm{~N})$ & $j_{5}(\mathrm{P})$ & $j_{\sigma}(\mathrm{K})$ & & $L w_{1}$ & $L w_{2}$ & \\
\hline 1 & 0.996143 & -0.02048 & 2.317871 & -0.37965 & -0.56815 & 0.54681 & -2.080196453 & 1.472587 & 0.699387 & -0.212174053 \\
\hline 2 & 0.838481 & 0.545057 & 1.347549 & 1.166583 & 0.741616 & 0.673065 & -1.217495956 & -0.60922 & 0.526448 & 0.540334675 \\
\hline 3 & -0.88541 & 1.155593 & -0.07306 & -0.36007 & 0.91443 & 0.29891 & -0.874658689 & -0.28682 & 1.10661 & \\
\hline 4 & -0.78139 & 1.617126 & -0.09689 & 1.246001 & 0.545242 & 0.535554 & 1.953430407 & 0.985855 & -1.55521 & \\
\hline 5 & 1.154236 & 1.305964 & -0.34628 & 1.030944 & -1.48709 & -1.23566 & -0.164765862 & -0.18309 & -0.66367 & \\
\hline 6 & -1.56626 & 3.327984 & 1.205375 & 0.906212 & -2.31703 & 1.897674 & 0.667870142 & -0.55526 & 0.804189 & \\
\hline 7 & 0.275443 & 1.076501 & -1.06431 & 1.218909 & 0.808466 & -0.84648 & 1.880952801 & -0.10458 & 1.113067 & \\
\hline 8 & 2.092393 & 0.073101 & -0.1403 & 0.38235 & -0.49332 & 0.539668 & 2.641857041 & 0.501492 & 0.781672 & \\
\hline
\end{tabular}

2 